\title{
The Propagation of Thermoelastic Waves in Anisotropic Media of Orthorhombic, Hexagonal, and Tetragonal Syngonies
}

\author{
Nurlybek A. Ispulov, ${ }^{1}$ Abdul Qadir, ${ }^{2}$ Marat Zhukenov, ${ }^{1}$ and Erkin Arinov $^{3}$ \\ ${ }^{1}$ S. Toraighyrov Pavlodar State University, Pavlodar 140008, Kazakhstan \\ ${ }^{2}$ Department of Electrical Engineering, Sukkur IBA University, Sindh, Pakistan \\ ${ }^{3}$ O. A. Baykonurov Zhezkazgan University, Zhezkazgan 100600, Kazakhstan
}

Correspondence should be addressed to Abdul Qadir; aqadir@iba-suk.edu.pk

Received 2 August 2017; Accepted 18 October 2017; Published 16 November 2017

Academic Editor: John D. Clayton

Copyright (C) 2017 Nurlybek A. Ispulov et al. This is an open access article distributed under the Creative Commons Attribution License, which permits unrestricted use, distribution, and reproduction in any medium, provided the original work is properly cited.

\begin{abstract}
The investigation of wave propagation in elastic medium with thermomechanical effects is bound to have important economic implications in the field of composite materials, seismology, geophysics, and so on. In this article, thermoelastic wave propagation in anisotropic mediums of orthorhombic and hexagonal syngony having heterogeneity along $z$-axis is studied. Such medium has second-order axis symmetry. By using analytical matriciant method, a set of equations of motions in thermoelastic medium are reduced to an equivalent set of the first-order differential equations. In the general case, for the given set of equations, structures of fundamental solutions are made and dispersion relations are obtained.
\end{abstract}

\section{Introduction}

The interaction between thermal and mechanical fields in solid bodies is studied by dynamical theory of thermoelasticity. This study has vast application in different branches of engineering, such as earthquake engineering, soil mechanics, aeronautics, and nuclear reactors.

The theory of classical thermoelasticity [1-6] is based on the supposition of heat conduction law proposed by Fourier, in which the temperature distribution is governed by a parabolic-type partial differential equation. According to this law a thermal perturbation is instantaneously felt everywhere inside a body. But physically it is unrealistic specifically in case of short-time responses. In order to take into account this discrepancy, generalized thermoelasticity has been developed based on modified Fourier law, in which the temperature distribution is governed by a hyperbolictype equation; consequently the heat transfer in solids is considered as a wave phenomenon instead of a diffusion phenomenon.

In order to analytically investigate the propagation of wave in anisotropic inhomogeneous medium a novel matriciant method has been developed. The method of matriciant allows studying wave propagation in anisotropic medium with various mechanical and physical properties [7].

The structures of matriciant for propagation of waves in infinite and finite periodical inhomogeneous media are investigated in various studies.

Verma analytically investigated problem propagation of thermoelastic wave along arbitrary direction in a heat conducting plate by employing the normal mode expansion method, available in generalized theory of thermoelasticity having single thermal relaxation time [8].

By combining matriciant method with linear transformation formation, the interaction between free harmonic waves and multilayered media is studied [9]. The solutions so obtained give dispersion characteristics of multilayered media.

The matriciant method has also been employed to analytically investigate the problems of reflection and refraction of waves on the interface of homogenous anisotropic thermoelastic mediums [10].

\section{A Matriciant Method}

Nowadays the solution of wide range of theoretical and applied problems in continuum dynamics requires more 
TABLE 1

\begin{tabular}{|c|c|c|c|c|}
\hline Crystal system & Characteristic symmetry & Syngony & Unit cell parameters & Independent parameters \\
\hline Triclinic & 1x 1-fold & -1 & $a \neq b \neq c ; \alpha \neq \beta \neq \gamma$ & 6 \\
\hline Monoclinic & 1x 2-fold & $2 / m$ & $a \neq b \neq c ; \alpha=\gamma=90^{\circ} ; \beta \neq 90^{\circ}$ & 4 \\
\hline Orthorhombic & $3 \times 2$-fold & $\mathrm{mmm}$ & $a \neq b \neq c ; \alpha=\beta=\gamma=90^{\circ}$ & 3 \\
\hline Tetragonal & 1x 4-fold & $4 / \mathrm{mmm}$ & $a=b \neq c ; \alpha=\beta=\gamma=90^{\circ}$ & 2 \\
\hline Trigonal & 1x 3-fold & $\begin{array}{c}6 / m m m(\mathrm{P}) \\
-3 m(\mathrm{R})\end{array}$ & $a=b \neq c ; \alpha=\beta=90^{\circ} ; \gamma=120^{\circ}$ & 2 \\
\hline Hexagonal & 1x 6-fold & $6 / \mathrm{mmm}$ & $a=b \neq c ; \alpha=\beta=90^{\circ} ; \gamma=120^{\circ}$ & 2 \\
\hline Cubic & 4x 3-fold & $m-3 m$ & $a=b=c ; \alpha=\beta=\gamma=90^{\circ}$ & 1 \\
\hline
\end{tabular}

thorough consideration of anisotropy and physical and mechanical properties. The main issue that appears in analyzing wave propagation in anisotropic medium is inapplicability of physical interpretations and mathematical methods developed for isotropic medium. The main reason is that it is not possible to separate waves into forward and backward moving waves. In addition, another difficulty is an existence of a lot of physical parameters.

The analytical investigation for the wave propagation is based on developing matrix techniques.

The main idea is to deduce initial equations of the continuous medium and equations describing wave propagation in medium; then by employing separation of variables method (solutions are represented as plane waves) these equations reduced to the equivalent set of ordinary differential equations with variable coefficients; the solutions so obtained are represented by plane waves. After that, the structure of a matriciant is built.

The advantage of a matriciant method is that it equally describes the wave propagation in the presence of one or several physical effects, such as thermoelastic, magnetoelastic, and piezoelastic and magnetoelectric, piezomagnetic, and magnetoelectric effects.

The structures of equations of motion in inhomogeneous medium are already defined [11-13]. In this way great analytical tools have been developed for the investigation of wave propagation behavior in inhomogeneous anisotropic medium.

\section{The Concept of Syngony}

Syngony is the crystallographic division of crystals based on unit cell configuration. It is the relation between the edges $a, b, c$ of a cell and angles $\alpha, \beta, \gamma$ between them. Being an important classification division in symmetry of crystals, each syngony includes several point symmetry groups, Bravais lattices, and spatial symmetry groups.

Combining a lattice with the different orders of rotation symmetry leads to the seven possible crystal systems. The lattices of the seven crystal systems consist of an infinite array of identical points each with an identical environment. The point symmetry of this environment is known as the syngony of the lattice.
Seven possible syngonies that describe 7 crystal systems are shown in Table 1 [14].

\section{The Matrix Formulation of Thermoelastic Waves Propagation}

The simultaneous solution of equations of motion provides a way to study the thermoelastic wave propagation in anisotropic medium. These equations are written as

$$
\begin{aligned}
\sigma_{i j, j} & =\rho \ddot{U}_{i} \\
\lambda_{i j} \frac{\partial \theta}{\partial x_{j}} & =-q_{i} \\
\frac{\partial q_{i}}{\partial x_{i}} & =-i \omega \beta_{i j} \varepsilon_{i j}-i \omega \frac{c_{\varepsilon}}{T_{0}} \theta,
\end{aligned}
$$

where $\sigma_{i j}$ represents the components of stress tensor, $\rho$ is density of the medium, $\lambda_{i j}$ shows the components of thermal conductivity tensor, $q_{i}$ represents the components of heat flow vector, $\omega$ is the angular frequency, $\beta_{i j}$ are thermomechanical constants, $\beta_{i j}=\beta_{j i}, \varepsilon_{i j}$ is the strain tensor, $c_{\varepsilon}$ is specific heat at constant strain, and $\theta=T-T_{0}$ is increase in temperature, when deformation is small $\left|\theta / T_{0}\right| \ll 1$.

Duhamel-Neumann relation relates the physical and mechanical quantities by

$$
\sigma_{i j}=c_{i j k l} \varepsilon_{k l}-\beta_{i j} \theta .
$$

Here $c_{i j}$ are the elastic parameters, $c_{i j k l}=c_{j i k l}=c_{i j l k}=c_{k l i j}$, and $\varepsilon_{k l}$ is the Cauchy tensor for small deformations.

Equations (1)-(4) express relation between temperature and mechanical stress produced in the process; it is also function of the thermal field and deformation, considering them as independent variables.

Thus, equations (1)-(4) constitute a closed system of thermoelasticity equations, which describes the propagation of thermoelastic waves.

Based on the method of separation of variables in the case of a harmonic function of time

$$
\begin{aligned}
& {\left[U_{i}(x, y, z, t) ; \sigma_{i j}(x, y, z, t) ; \theta ; q_{z}\right]} \\
& \quad=\left[U_{i}(z), \sigma_{i j}(z), \theta ; q_{z}\right] e^{i(\omega t-m x-n y)} .
\end{aligned}
$$




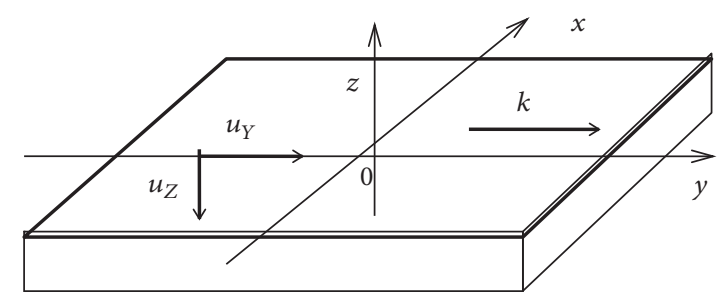

FIGURE 1: The coordinate system of the question of the wave propagation along the $z$-axis.

The system of equations (1)-(4) is reduced to a system of differential equations of first order with variable coefficients which describes the propagation of harmonic waves:

$$
\frac{d \vec{W}}{d z}=B \vec{W}
$$

Here $B=B\left\lfloor c_{i j k l}(z), \beta_{i j}(z), \omega, m, n\right\rfloor$ is coefficient matrix whose elements contain the information regarding the thermoelastic wave propagation in the medium and $m$ and $n$ represent the $x$ and $y$ components of the wave vector $\widetilde{\kappa}$, respectively.

The boundary condition column vector $\vec{W}$ has the form

$$
\begin{aligned}
\vec{W} & (x, y, z, t) \\
& =\left[u_{z}(z), \sigma_{z z}, u_{x}(z), \sigma_{x z}, u_{y}(z), \sigma_{y z}, \theta, q_{z}\right]^{t} \\
& \cdot \exp (i \omega t-i m x-i n y),
\end{aligned}
$$

where " $t$ " indicates the transpose of the vector.

Figure 1 schematically shows the unit cell of the crystal. A Cartesian coordinate system is shown in which the heterogeneity of the medium is assumed along the $z$-axis. Here $U x$, $U y$ are the components of the displacement vector $\mathbf{U}$ and $k$ is the wave vector.

For such case, in constructing the coefficient matrix, $B$ is used as a representation of the solution (5), the systems of equations (1)-(4) are in the derivatives along the coordinate $Z$, and the excluded components of the stress tensor are not incorporated in the boundary conditions. The multiplier $\exp (i \omega t-i m x-i n y)$ is omitted throughout.

Let us consider the construction of the set of first-order differential equations (6) for tetragonal syngony of classes $4, \overline{4}, 4 / \mathrm{m}$, which are described by fourfold axis symmetry parallel to $z$-axis. Seven elastic and three thermomechanical parameters are present in this case.

We consider that

$$
\begin{aligned}
& \frac{\partial f}{\partial z} \longrightarrow \frac{d f}{d z} \\
& \frac{\partial f}{\partial x} \longrightarrow-i k_{x} f
\end{aligned}
$$

$$
\begin{aligned}
& \frac{\partial f}{\partial y} \longrightarrow-i k_{y} f, \\
& \frac{\partial f}{\partial t} \longrightarrow i \omega f, \\
& m=k_{x}, \\
& n=k_{y} .
\end{aligned}
$$

For tetragonal syngony (classes $4, \overline{4}, 4 / \mathrm{m}$ ) DuhamelNeumann expression (4) can be written in matrix form as [6]

$$
\begin{aligned}
\left(\begin{array}{c}
\sigma_{x x} \\
\sigma_{y y} \\
\sigma_{z z} \\
\sigma_{y z} \\
\sigma_{x z} \\
\sigma_{x y}
\end{array}\right) \\
=\left(\begin{array}{cccccc}
c_{11} & c_{12} & c_{13} & 0 & 0 & c_{16} \\
c_{12} & c_{11} & c_{13} & 0 & 0 & -c_{16} \\
c_{13} & c_{13} & c_{33} & 0 & 0 & 0 \\
0 & 0 & 0 & c_{44} & 0 & 0 \\
0 & 0 & 0 & 0 & c_{44} & 0 \\
c_{16} & -c_{16} & 0 & 0 & 0 & c_{66}
\end{array}\right)\left(\begin{array}{l}
\varepsilon_{1} \\
\varepsilon_{2} \\
\varepsilon_{3} \\
\varepsilon_{4} \\
\varepsilon_{5} \\
\varepsilon_{6}
\end{array}\right) \\
-\left(\begin{array}{ccc}
\beta_{11} & 0 & \beta_{13} \\
0 & \beta_{11} & \beta_{13} \\
\beta_{13} & \beta_{13} & \beta_{33} \\
0 & 0 & 0 \\
0 & 0 & 0 \\
0 & 0 & 0
\end{array}\right)
\end{aligned}
$$

In (4) the following notations are used:

$$
\begin{aligned}
& \varepsilon_{1}=\varepsilon_{11}=U_{1,1}=\frac{\partial U_{x}}{\partial x}, \\
& \varepsilon_{2}=\varepsilon_{22}=U_{2,2}=\frac{\partial U_{y}}{\partial y},
\end{aligned}
$$




$$
\begin{aligned}
& \varepsilon_{3}=\varepsilon_{33}=U_{3,3}=\frac{\partial U_{z}}{\partial z} \\
& \varepsilon_{4}=2 \varepsilon_{23}=U_{2,3}+U_{3,2}=\frac{\partial U_{y}}{\partial z}+\frac{\partial U_{z}}{\partial y}, \\
& \varepsilon_{5}=2 \varepsilon_{21}=U_{1,3}+U_{3,1}=\frac{\partial U_{x}}{\partial z}+\frac{\partial U_{z}}{\partial x}, \\
& \varepsilon_{6}=2 \varepsilon_{12}=U_{1,2}+U_{2,1}=\frac{\partial U_{x}}{\partial y}+\frac{\partial U_{y}}{\partial x} .
\end{aligned}
$$

Components of the mechanical stress tensor in DuhamelNeumann expression, for the given class of crystals, will take the following form:

$$
\begin{aligned}
\sigma_{x x}= & -c_{11} i m U_{x}-c_{12} i n U_{y}+c_{13} \frac{d U_{z}}{d Z} \\
& -c_{16}\left(i n U_{x}+i m U_{y}\right)-\left(\beta_{11}+\beta_{13}\right) \theta \\
\sigma_{y y}= & -c_{12} i m U_{x}-c_{11} i n U_{y}+c_{13} \frac{d U_{z}}{d Z} \\
& +c_{16}\left(i n U_{x}+i m U_{y}\right)-\left(\beta_{11}+\beta_{13}\right) \theta \\
\sigma_{z z}= & -c_{13} i m U_{x}-c_{13} i n U_{y}+c_{33} \frac{d U_{z}}{d Z}-\left(2 \beta_{13}+\beta_{33}\right) \theta \\
\sigma_{y z}= & c_{44}\left(\frac{d U_{y}}{d Z}-i n U_{z}\right) \\
\sigma_{x z}= & c_{44}\left(\frac{d U_{x}}{d Z}-i m U_{z}\right) \\
\sigma_{x y}= & c_{16} i n U_{y}-c_{16} i m U_{x}-c_{66}\left(i n U_{x}+i m U_{y}\right) .
\end{aligned}
$$

The following notations are introduced for relationship (10):

$$
\begin{aligned}
& \varepsilon_{1}=\varepsilon_{11}=U_{1,1}, \\
& S_{2}=\varepsilon_{22}=U_{2,2}, \\
& S_{3}=\varepsilon_{33}=U_{3,3} \\
& S_{4}=2 \varepsilon_{23}=U_{2,3}+U_{3,2}, \\
& S_{5}=2 \varepsilon_{21}=U_{1,3}+U_{3,1}, \\
& S_{6}=2 \varepsilon_{12}=U_{1,2}+U_{2,1} .
\end{aligned}
$$

From (10) let us express derivatives of the displacement vector $\vec{U}$ with respect to $z$ component:

$$
\begin{aligned}
& \frac{d U_{z}}{d Z}=\frac{1}{c_{33}} \sigma_{z z}+\frac{c_{13}}{c_{33}} i m U_{x}+\frac{c_{13}}{c_{33}} i n U_{y}+\frac{2 \beta_{13}+\beta_{33}}{c_{33}} \theta \\
& \frac{d U_{y}}{d Z}=\frac{1}{c_{44}} \sigma_{y z}+i n U_{z} \\
& \frac{d U_{x}}{d Z}=\frac{1}{c_{44}} \sigma_{z x}+i m U_{z} .
\end{aligned}
$$

From (1), and also taking into account (10), we can get the following relationships:

$$
\begin{aligned}
& \frac{d \sigma_{x z}}{d Z} \\
& =\frac{c_{13}}{c_{33}} i m \sigma_{z z} \\
& +\left[-\rho \omega^{2}+m^{2}\left(c_{11}-\frac{c_{13}^{2}}{c_{33}}\right)+c_{16} m n+c_{66} n^{2}\right] U_{x} \\
& + \\
& \quad+\frac{c_{13}}{c_{33}}\left(2 \beta_{13}+\beta_{33}\right) i m \theta \\
& \left.\left.\frac{d \sigma_{y z}}{d Z} \quad \frac{c_{13} c_{23}}{c_{33}}\right)+c_{16} n^{2}\right] U_{y} \\
& =\frac{c_{13}}{c_{33}} i n \sigma_{z z}+m n\left[c_{12}+c_{66}-\frac{c_{13}^{2}}{c_{33}}\right] U_{x} \\
& +\left(-\rho \omega^{2}+c_{66} m^{2}-c_{16} m n+\left(c_{11}-\frac{c_{13}^{2}}{c_{33}}\right) n^{2}\right) U_{y} \\
& +\frac{c_{23}}{c_{33}}\left(2 \beta_{13}+\beta_{33}\right) i n \theta \\
& \frac{d \sigma_{z z}}{d Z}=-\rho \omega^{2} U_{z}+i m \sigma_{x z}+i n \sigma_{y z} \cdot \\
& +
\end{aligned}
$$

Fourier thermal conductivity equation (2) for anisotropic medium in the component form can be written as

$$
\begin{aligned}
& -q_{x}=\lambda_{11} \frac{\partial \theta}{\partial x}+\lambda_{12} \frac{\partial \theta}{\partial y}+\lambda_{13} \frac{\partial \theta}{\partial z} \\
& -q_{y}=\lambda_{12} \frac{\partial \theta}{\partial x}+\lambda_{22} \frac{\partial \theta}{\partial y}+\lambda_{23} \frac{\partial \theta}{\partial z} \\
& -q_{z}=\lambda_{13} \frac{\partial \theta}{\partial x}+\lambda_{23} \frac{\partial \theta}{\partial y}+\lambda_{33} \frac{\partial \theta}{\partial z}
\end{aligned}
$$

Given set in a matrix form is

$$
\left(\begin{array}{c}
-q_{x} \\
-q_{y} \\
-q_{z}
\end{array}\right)=\left(\begin{array}{lll}
\lambda_{11} & \lambda_{12} & \lambda_{13} \\
\lambda_{12} & \lambda_{22} & \lambda_{23} \\
\lambda_{13} & \lambda_{23} & \lambda_{33}
\end{array}\right)\left(\begin{array}{c}
-i m \theta \\
-i n \theta \\
\frac{d \theta}{d z}
\end{array}\right)
$$

Consequently, from (2) we get equation for $d \theta / d z$ :

$$
\frac{d \theta}{d z}=-\frac{1}{\lambda_{33}} q_{z}+\left(\frac{\lambda_{13}}{\lambda_{33}} i m+\frac{\lambda_{23}}{\lambda_{33}} i n\right) \theta .
$$


From the heat input equation (3) with relationships $(2)^{\prime},(14)$, and first equation of (12), we get the following relationship:

$$
\begin{aligned}
\frac{d q_{z}}{d Z}= & -i \omega \frac{2 \beta_{13}+\beta_{33}}{c_{33}} \sigma_{z z}+\omega m \frac{c_{13}}{c_{33}}\left(2 \beta_{13}+\beta_{33}\right) U_{x} \\
& +\omega n \frac{c_{23}}{c_{33}}\left(2 \beta_{13}+\beta_{33}\right) U_{y}-i \omega\left(\frac{c_{\varepsilon}}{T_{0}}+\frac{\beta_{33}^{2}}{c_{11}}\right) \theta \\
& +\left(\frac{\lambda_{13}}{\lambda_{33}} i m+\frac{\lambda_{23}}{\lambda_{33}} i n\right) q_{z} .
\end{aligned}
$$

As a result, for the tetragonal syngony of $4, \overline{4}, 4 / \mathrm{m}$ classes in case of thermoelastic waves propagation the set of differential equations of the first order takes the following form:

$$
\begin{aligned}
& \frac{d U_{z}}{d Z}=\frac{1}{c_{33}} \sigma_{z z}+\frac{c_{13}}{c_{33}} i m U_{x}+\frac{c_{13}}{c_{33}} i n U_{y}+\frac{2 \beta_{13}+\beta_{33}}{c_{33}} \theta \\
& \frac{d \sigma_{z z}}{d Z}=-\rho \omega^{2} U_{z}+i m \sigma_{x z}+i n \sigma_{y z} \\
& \frac{d U_{x}}{d Z}=\frac{1}{c_{44}} \sigma_{z x}+i m U_{z} \\
& \frac{d \sigma_{x z}}{d Z} \\
& =\frac{c_{13}}{c_{33}} i m \sigma_{z z} \\
& +\left[-\rho \omega^{2}+m^{2}\left(c_{11}-\frac{c_{13}^{2}}{c_{33}}\right)+c_{16} m n+c_{66} n^{2}\right] U_{x} \\
& +\left[m n\left(c_{12}+c_{66}-\frac{c_{13} c_{23}}{c_{33}}\right)+c_{16} n^{2}\right] U_{y} \\
& +\frac{c_{13}}{c_{33}}\left(2 \beta_{13}+\beta_{33}\right) i m \theta \\
& \frac{d U_{y}}{d Z}=\frac{1}{c_{44}} \sigma_{y z}+i n U_{z} \\
& \frac{d \sigma_{y z}}{d Z} \\
& =\frac{c_{13}}{c_{33}} i n \sigma_{z z}+m n\left[c_{12}+c_{66}-\frac{c_{13}^{2}}{c_{33}}\right] U_{x} \\
& +\left(-\rho \omega^{2}+c_{66} m^{2}-c_{16} m n+\left(c_{11}-\frac{c_{13}^{2}}{c_{33}}\right) n^{2}\right) U_{y} \\
& +\frac{c_{23}}{c_{33}}\left(2 \beta_{13}+\beta_{33}\right) i n \theta \\
& \frac{d \theta}{d z}=-\frac{1}{\lambda_{33}} q_{z}+\left(\frac{\lambda_{13}}{\lambda_{33}} i m+\frac{\lambda_{23}}{\lambda_{33}} i n\right) \theta
\end{aligned}
$$

$$
\begin{aligned}
& \frac{d q_{z}}{d Z} \\
& =-i \omega \frac{2 \beta_{13}+\beta_{33}}{c_{33}} \sigma_{z z}+\omega m \frac{c_{13}}{c_{33}}\left(2 \beta_{13}+\beta_{33}\right) U_{x} \\
& \quad+\omega n \frac{c_{23}}{c_{33}}\left(2 \beta_{13}+\beta_{33}\right) U_{y}-i \omega\left(\frac{c_{\varepsilon}}{T_{0}}+\frac{\beta_{33}^{2}}{c_{11}}\right) \theta \\
& \quad+\left(\frac{\lambda_{13}}{\lambda_{33}} i m+\frac{\lambda_{23}}{\lambda_{33}} i n\right) q_{z} .
\end{aligned}
$$

From this set of equations (6)' the expression for the coefficient of matrix $B$ can be derived.

The structures of the matrix $B$ and column vector $W$ boundary condition in case of bulk orthorhombic, hexagonal, and tetragonal crystal system having second-order axis of symmetry and heterogeneity along the $z$-axis are given by

B

$$
\begin{aligned}
& =\left[\begin{array}{cccccccc}
0 & b_{12} & b_{13} & 0 & b_{15} & 0 & b_{17} & 0 \\
b_{21} & 0 & 0 & b_{24} & 0 & b_{26} & 0 & 0 \\
b_{24} & 0 & 0 & b_{34} & 0 & 0 & 0 & 0 \\
0 & b_{13} & b_{43} & 0 & b_{45} & 0 & b_{47} & 0 \\
b_{26} & 0 & 0 & 0 & 0 & b_{56} & 0 & 0 \\
0 & b_{15} & b_{45} & 0 & b_{65} & 0 & b_{67} & 0 \\
0 & 0 & 0 & 0 & 0 & 0 & 0 & b_{78} \\
0 & -i \omega b_{17} & -i \omega b_{47} & 0 & -i \omega b_{67} & 0 & b_{87} & 0
\end{array}\right] ; \\
& \vec{W}=\left(\begin{array}{c}
u_{z} \\
\sigma_{z z} \\
u_{x} \\
\sigma_{x z} \\
u_{y} \\
\sigma_{y z} \\
\theta \\
q_{z}
\end{array}\right) .
\end{aligned}
$$

From the structure of the coefficient matrix as given in (16) it is revealed that, in the spatial case, the elastic waves of different polarization and the heat wave are interrelated.

The nonzero elements $b_{13}, b_{24}$ of the matrix $B$ determine the mutual transformation of longitudinal and transverse waves polarized along $x$-axis, whereas elements $b_{15}, b_{26}$ describe the relationship between transverse waves polarized along $y$-axis and longitudinal wave. Nonzero element $b_{45}$ defines the mutual transformation between the waves of transverse polarization.

The fact that

$$
b_{17}=\frac{2 \beta_{13}+\beta_{33}}{c_{33}}
$$


means that the longitudinal wave is generated due to thermoelastic effect.

Nonzero elements $b_{47}$ and $b_{67}$ given as

$$
\begin{aligned}
& b_{47}=\frac{c_{13}}{c_{33}}\left(2 \beta_{13}+\beta_{33}\right) i m ; \\
& b_{67}=\frac{c_{23}}{c_{33}}\left(2 \beta_{13}+\beta_{33}\right) i n
\end{aligned}
$$

indicate the effect of transversely polarized elastic wave on thermoelastic effect, whereas the effect of thermoelasticity on elastic shear wave having X-polarization is described by $b_{47}$, while the effect of thermoelasticity on transverse polarized wave is given by $b_{67}$.

Similarly, for the thermoelastic waves propagating in an anisotropic medium of cubic symmetry the coefficient matrix is constructed for the bulk case and the analysis of matrix coefficients has been carried out. We also obtain the structure of the coefficient matrix in the case of thermoelastic waves propagation in anisotropic media, orthorhombic, tetragonal, hexagonal, and cubic crystal systems.

\section{Structure of Matriciant}

Normalized solution of (6) is called matriciant. Any other solution that has meaning of the matrix of fundamental solutions has the following form $[15,16]$ :

$$
\widehat{X}=\widehat{T}\left(z_{0}, z\right) \widehat{C},
$$

where $\widehat{T}\left(z_{0}, z\right)$ represents matriciant and $\widehat{C}$ shows some constant matrix.

Set of equations (6) can be written as

$$
\vec{W}=\widehat{T} \vec{W}_{0}
$$

where $T$ is matrix of fundamental solutions and $\vec{W}_{0}$ is boundary condition vector, defined by conditions at $z=z_{0}$; then

$$
\frac{d \vec{W}_{0}}{d z}=\frac{d \widehat{T}}{d z} \vec{W}_{0}=\widehat{B} \widehat{T} \vec{W}_{0}
$$

Consequently, as a result of $\vec{W}_{0}$ being an arbitrary vector, the matriciant satisfies equation (6):

$$
\frac{d \widehat{T}}{d z}=\widehat{B} \widehat{T}
$$

Normalized solution of (6) can be made by the method of successive approximations from the recurrence relations:

$$
\frac{d \widehat{T}_{k}}{d z}=\widehat{B} \widehat{T}_{k-1} \text {. }
$$

Assuming that $\widehat{T}_{k}\left(z_{0}\right)=\widehat{E}(k=0,1,2, \ldots)$ for $\widehat{T}_{k}$ we get

$$
\widehat{T}_{k}=\widehat{E}+\int_{z_{0}}^{z} \widehat{B}\left(z_{1}\right) \widehat{T}_{k-1}\left(z_{1}\right) d z_{1} .
$$

Consequently, we get

$T_{0}=E$,

$$
\begin{aligned}
T_{1}= & E+\int_{z_{0}}^{z} B\left(z_{1}\right) d z_{1} \\
T_{2}= & E+\int_{z_{0}}^{z} B\left(z_{1}\right) d z_{1}+\int_{z_{0}}^{z} \int_{z_{0}}^{z_{1}} B\left(z_{1}\right) B\left(z_{2}\right) d z_{1} d z_{2} \\
& +\cdots
\end{aligned}
$$

In (25) $\dot{T}$ is represented in the form of infinite matrix series:

$$
\begin{aligned}
T= & E+\int_{0}^{z} B d z_{1}+\int_{0}^{z} \int_{0}^{z_{1}} B\left(z_{1}\right) B\left(z_{2}\right) d z_{1} d z_{2} \\
& +\cdots
\end{aligned}
$$

For the matriciant, the following relations are satisfied [9]:

(1) $T\left(z_{0}, z\right)=T\left(z_{1}, z\right) T\left(z_{0}, z_{1}\right)$.

(2) $\ln \left|T\left(z_{0}, z\right)\right|=\int_{z_{0}}^{z} \operatorname{spB}\left(z_{1}\right) d z_{1}$.

(3) If $B=B_{0}$ which represents a constant matrix, to $T_{0}=$ $\exp \left[B_{0}\left(z-z_{0}\right)\right]$

(4) $d T^{-1} / d z=-T^{-1} B$.

The fourth property of the matriciant gives representation in the form of infinite matrix series for the inverse matriciant. Assuming the same as we did to get (26)

$$
\begin{aligned}
& T_{0}^{-1}=E \\
& T_{k}^{-1}=E-\int_{z_{0}}^{z} T_{k-1}\left(z_{1}\right) B\left(z_{1}\right) d z_{1}
\end{aligned}
$$

we have

$$
\begin{aligned}
T_{1}^{-1}= & E-\int_{z_{0}}^{z} B\left(z_{1}\right) d z_{1} \\
T_{2}^{-1}= & E-\int_{z_{0}}^{z} B\left(z_{1}\right) d z_{1} \\
& +\int_{z_{0}}^{z} \int_{z_{0}}^{z_{1}} B\left(z_{2}\right) B\left(z_{1}\right) d z_{1} d z_{2},
\end{aligned}
$$

from which the desired form of $T^{-1}$ is obtained and given as

$$
T^{-1}=E-\int_{0}^{z} B d z_{1}+\int_{0}^{z} \int_{0}^{z_{1}} B\left(z_{2}\right) B\left(z_{1}\right) d z_{1} d z_{2}
$$

Both series converge on (26) and (29) absolutely and uniformly on any finite interval in which the elements of the matrix $B(z)$ are continuous.

Let

$$
\begin{aligned}
& g(z)=\max \left|b_{i j}\right|, \\
& h(z)=\left|\int_{z_{0}}^{z} g(z) d z\right| .
\end{aligned}
$$


Each of $n^{2}$ scalar series is dominated by the series

$$
1+h(z)+\frac{n h^{2}}{2 !}+\frac{n^{2} h^{2}}{3 !} .
$$

Matrix series (26) and (29) is the sum of matrices

$$
\begin{aligned}
T & =\sum_{n=0}^{\infty} T_{(n)}, \\
T^{-1} & =\sum_{n=0}^{\infty} T_{(n)}^{-1} .
\end{aligned}
$$

Index $(n)$ coincides with the number of matrices $B\left(z_{i}\right)$ multiplied in the integrand.

In this case, formation of the matriciant structure is to set relation between elements of $T$ and $T^{-1}$.

In this case, the relation

$$
T T^{-1}=T^{-1} T=E
$$

Building structure of matriciant establishes relationship between elements of the direct and inverse matrices $T$ and $T^{-1}$ based on comparison of their piecemeal approach.

Endless rows of the matrix can be written as

$$
\begin{gathered}
T=T_{\ell}+T_{o}, \\
T^{-1}=T_{\ell}^{-1}-T_{o}^{-1},
\end{gathered}
$$

where $T_{e, o}^{ \pm}$is the sum of even and odd rows (26) and (29).

By induction we prove that the structure of $T^{-1}(2 n)$ and $T^{-1}(2 n+1)$ is stored for each $n$.

Structure matriciant, in the case of propagation of thermoelastic waves in cubic, hexagonal, and orthorhombic crystal systems in the bulk case, is defined as

$$
\begin{aligned}
& T^{-1} \\
& =\left(\begin{array}{cccccccc}
t_{22} & -t_{12} & -t_{42} & t_{32} & -t_{62} & t_{52} & -t_{82} & t_{72} \\
-t_{21} & t_{11} & t_{41} & -t_{31} & t_{61} & -t_{51} & t_{81} & -t_{71} \\
-t_{24} & t_{14} & t_{44} & -t_{34} & t_{64} & -t_{54} & t_{84} & -t_{74} \\
t_{23} & -t_{13} & -t_{43} & t_{33} & -t_{63} & t_{53} & -t_{83} & t_{73} \\
-t_{26} & t_{16} & t_{46} & -t_{36} & t_{66} & -t_{56} & t_{86} & -t_{76} \\
t_{25} & -t_{15} & -t_{45} & t_{35} & -t_{65} & t_{55} & -t_{85} & t_{75} \\
-t_{28} & t_{18} & t_{48} & -t_{38} & t_{68} & -t_{58} & t_{88} & -t_{78} \\
t_{27} & -t_{17} & -t_{47} & t_{37} & -t_{67} & t_{57} & -t_{87} & -t_{77}
\end{array}\right) .
\end{aligned}
$$

Elements tij of matriciant $T^{-1}$ are elements of direct matriciant $T$.

We obtain the structure of the matriciant representing thermoelastic waves propagation in these classes in the $X Z$ and $Y Z$ plane, respectively.
In one case (propagation along the $z$-axis $(m=0, n=0))$, structure (35) takes the following form:

$$
\begin{aligned}
T^{-1} & =\left(\begin{array}{cccc}
t_{22} & -t_{12} & -t_{82} & t_{72} \\
-t_{21} & t_{11} & t_{81} & -t_{71} \\
-t_{28} & t_{18} & t_{88} & -t_{78} \\
t_{27} & -t_{17} & -t_{87} & t_{77}
\end{array}\right) ; \\
T^{-1} & =\left(\begin{array}{cc}
t_{44} & -t_{34} \\
-t_{43} & t_{33}
\end{array}\right) ; \\
T^{-1} & =\left(\begin{array}{cc}
t_{66} & -t_{56} \\
-t_{65} & t_{55}
\end{array}\right) .
\end{aligned}
$$

Building matriciant structures, in this case, is basically making the relationships between elements of the direct and inverse matrices $T$ and $T^{-1}$ based on comparison of their piecemeal approach.

Decomposition of the structure of $(8 \times 8)$ matrix as given in $(35)$ into a $(4 \times 4)$ matrix and two $(2 \times 2)$ matrix means the independence of the elastic longitudinal wave with a thermal effect and the elastic shear wave. At the same time, the elastic shear waves, with one-dimensional propagation in anisotropic media, cubic, hexagonal, and orthorhombic crystal systems, along the symmetry axis of even order are also obtained without the thermoelastic effect.

\section{Dispersion Equations for the Elastic and Thermoelastic Anisotropic Mediums}

The main characteristics that determine the patterns of propagation of wave in an unbounded periodic structure are dispersion equations. The dispersion relations are the following relationships: $\nu=\nu(\omega), \kappa=\kappa(\omega), \omega=\omega(\kappa)$, and $\omega=\omega(\nu)$, where $\nu$ is the speed, $\omega$ is the cyclic frequency, and $\kappa$ is wave vector. In the particular case we obtain the dependence $\kappa=\kappa(\omega)$. The resulting structure being higher than matriciant allows one to modify the condition of existence of nontrivial solutions and to lower twice the degree of the characteristic equation.

It follows from Bloch's theorem that when periodic inhomogeneity is present, $\vec{W}$ vector can have the form

$$
\vec{W}(h)=e^{i \tilde{k} h} \vec{W}(0)
$$

where $\vec{W}$ is column vector which is a solution to the set of equations (6).

On the other hand, using monodromy matrix (i.e., matriciant of (6) for one period) we have

$$
\vec{W}(h)=\widehat{T} \vec{W}(0)
$$


Combining (37) and (38), we get

$$
\left(\widehat{T}-e^{i k h} E\right) \vec{W}(0)=0
$$

From now on, $E$ will represent identity matrix. From the condition

$$
\operatorname{det}\left[T-e^{i k h} E\right]=0
$$

follows secular equation, whose roots define desired dispersion equations.

Let us multiply (39) and $T^{-1} e^{-i \tilde{k} h}$. It is possible when $\operatorname{det} T=1$ since $\left|e^{ \pm i k h}\right|=1$. As a result, (39) takes the following form:

$$
\left(T^{-1}-e^{-i \vec{k} h}\right) \vec{W}(0)=0
$$

Relationships (39) and (41) are equivalent and they define the same spectrum. Physically, this means that waves propagate in opposite directions in the medium that has infinite structure with the same dispersion pattern. Combining (39) and (41), we obtain the next modified form for condition of existence of nontrivial solutions

$$
\operatorname{det}[p-E \cos \widetilde{k} h]=0 \text {, }
$$

where

$$
p=\frac{1}{2}\left[T+T^{-1}\right]
$$

and obtain the dispersion equation of thermoelastic waves propagating in anisotropic media, cubic, hexagonal, and orthorhombic crystal systems in the bulk case, having the following form:

$$
\begin{aligned}
& \cos \widetilde{k}_{1} h=-\frac{a}{4}-\frac{1}{4 \sqrt{3}}\left(\sqrt{3 \sqrt[3]{\gamma}+3 a^{2}-8 b+\frac{5\left(b^{2}-3 a c+12 d\right)}{\sqrt[3]{\gamma}}}\right) \\
& -\frac{1}{5}\left(\sqrt{-\sqrt[3]{\gamma}+3 a^{2}-8 b+\frac{2\left(b^{2}-3 a c+12 d\right)}{\sqrt[3]{\gamma}}+\frac{5\left(a^{3}-4 b a+8 c\right)}{3 \sqrt[3]{\gamma}}+3 a^{2}-8 b}\right) \\
& \cos \tilde{k}_{2} h=-\frac{a}{4}-\frac{1}{4}\left(\sqrt{3 \sqrt[3]{\gamma}+3 a^{2}-8 b+\frac{5\left(b^{2}-3 a c+12 d\right)}{\sqrt[3]{\gamma}}}\right) \\
& +\frac{1}{5}\left(\sqrt{-\sqrt[3]{\gamma}+3 a^{2}-8 b+\frac{2\left(b^{2}-3 a c+12 d\right)}{\sqrt[3]{\gamma}}+\frac{5\left(a^{3}-4 b a+8 c\right)}{3 \sqrt[3]{\gamma}}+3 a^{2}-8 b}\right) \\
& \cos \widetilde{k}_{3} h=-\frac{a}{4}+\frac{1}{4}\left(\sqrt{3 \sqrt[3]{\gamma}+3 a^{2}-8 b+\frac{5\left(b^{2}-3 a c+12 d\right)}{\sqrt[3]{\gamma}}}\right) \\
& -\frac{1}{5}\left(\sqrt{-\sqrt[3]{\gamma}+3 a^{2}-8 b+\frac{2\left(b^{2}-3 a c+12 d\right)}{\sqrt[3]{\gamma}}+\frac{5\left(a^{3}-4 b a+8 c\right)}{3 \sqrt[3]{\gamma}}+3 a^{2}-8 b}\right) \\
& \cos \widetilde{k}_{4} h=-\frac{a}{4}+\frac{1}{4}\left(\sqrt{3 \sqrt[3]{\gamma}+3 a^{2}-8 b+\frac{5\left(b^{2}-3 a c+12 d\right)}{\sqrt[3]{\gamma}}}\right) \\
& +\frac{1}{5}\left(\sqrt{-\sqrt[3]{\gamma}+3 a^{2}-8 b+\frac{2\left(b^{2}-3 a c+12 d\right)}{\sqrt[3]{\gamma}}-\frac{5\left(a^{3}-4 b a+8 c\right)}{3 \sqrt[3]{\gamma}}+3 a^{2}-8 b}\right)
\end{aligned}
$$


where

$$
\gamma=\sqrt[3]{2 b^{3}-9(a c+8 d) b+27\left(c^{2}+a^{2} d\right)+\sqrt{\left(2 b^{3}-9(a c+8 d) b+27\left(d a^{2}+c^{2}\right)\right)^{2}-4\left(b^{2}-3 a c+12 d\right)^{3}}} .
$$

$a, b, c$ show the elements of matrix (43).

These dispersion equations are obtained using the mathematical package Mathematica 8.0.

Knowledge of the monodromy matrix in an analytical form allows obtaining matriciant of arbitrary periodic inhomogeneous layer.

If there are $n$ periods, sequence of equations is as follows:

$$
\begin{aligned}
& \vec{u}_{1}=T \vec{u}_{0}, \\
& \vec{u}_{2}=T \vec{u}_{1}, \ldots, \vec{u}_{n}=T \vec{u}_{n-1}
\end{aligned}
$$

which leads to

$$
\vec{u}_{n}=T^{n} \vec{u}_{0} .
$$

Thus, calculating the matriciant for periodically inhomogeneous layer having $n$ periods is associated with the calculation of $n$th power of the monodromy matrix.

The introduction of regular structures is important for the matrix $p$ (43) which gives the recurrence relation:

$$
T^{2}=2 p T-E .
$$

Consistent application of (48) allows us to obtain $T^{n}$ as follows:

$$
T^{n}=P_{n}(p) T-P_{n-1}(p),
$$

where $P_{n}(p)$ represents matrix Chebyshev-Gegenbauer polynomials.

\section{Conclusion}

In this paper, the thermoelastic wave propagation in anisotropic mediums of orthorhombic and hexagonal syngony has been analytically investigated by using matriciant method. The system of first-order differential equation with variable coefficients is constructed by using separation of variable method. Coefficients matrix for all seven types of anisotropic mediums for one-, two-, and three-dimensional cases was obtained. The structure of fundamental solutions in the form of system of first-order differential equations that describes thermoelastic waves propagation in anisotropic mediums for one-, two-, and three-dimensional cases was formed. Variance equations of thermoelastic waves for unlimited periodic structure were also obtained. For fourthorder coefficients matrix, the problems of wave reflection and refraction on the border of uniform anisotropic thermoelastic mediums were solved analytically.

\section{Conflicts of Interest}

The authors declare that they have no conflicts of interest.

\section{References}

[1] W. Nowacki, Dynamic Problems of Thermoelasticity, Noordhoff, The Netherlands, 1975.

[2] W. Nowacki, Thermoelasticity, Pergamon Press, Oxford, UK, 2nd edition, 1986.

[3] N. A. Ispulov, A. Qadir, M. K. Zhukenov, T. S. Dossanov, and T. G. Kissikov, "The analytical form of the dispersion equation of elastic waves in periodically inhomogeneous medium of different classes of crystals," Advances in Mathematical Physics, vol. 2017, Article ID 5236898, 2017.

[4] A. L. Shuvalov, O. Poncelet, and M. Deschamps, "General formalism for plane guided waves in transversely inhomogeneous anisotropic plates," Wave Motion, vol. 40, no. 4, pp. 413-426, 2004.

[5] K. Tanuma, "Stroh formalism and rayleigh waves," Journal of Elasticity, vol. 89, no. 1-3, pp. 5-154, 2007.

[6] A. Morro and G. Caviglia, "Wave propagation in a stratified layer via the matricant," Communications in Applied and Industrial Mathematics, vol. 2, no. 1, 2011.

[7] S. Tleukenov, Matriciant method. - Pavlodar: PSU after S. Toraighyrov, p. 148, 2004.

[8] K. L. Verma, "Thermoelastic waves in anisotropic laminates with thermal relaxation," International Journal of Modern Mathematics, vol. 3, no. 1, pp. 75-87, 2008.

[9] K. L. Verma, "The general problem of thermoelastic wave propagation in multilayered anisotropic media withapplication toperiodic media," International Journal of Applied Engineering Research, vol. 1, no. 4, pp. 908-922, 2011.

[10] N. A. Ispulov, A. Qadir, and M. A. Shah, "Reflection of thermoelastic wave on the interface of isotropic half-space and tetragonal syngony anisotropic medium of classes $4,4 / \mathrm{m}$ with thermomechanical effect," Chinese Physics B, vol. 25, no. 3, Article ID 038102, 2016.

[11] Z. S. Erzhanov, N. Z. Zhubaev, and S. K. Tleukenov, "Seismic waves in an inhomogeneous medium," Gerlands Beitrage zur Geophysik, vol. 94, no. 1, pp. 45-51, 1985.

[12] S. Tleykenov, The structure of propagator matrix and it is application in the case of the periodical inhomogeneous media. Abstr. Semin. on Earthquake processes and their consequences Seismological investigations. 1989. Kurukshetra, India. P. 2-4.

[13] S. Tleykenov, Investigation of the thin layer influence of the boundary conditions. Abstacts "Seminar on eastquare processe and their consequences”. 1989. Kurukshetra, India. P. 4.

[14] N. Ashkroft and N. Mermin, Solid state physics. Vol 1. Chapter 8, 2004.

[15] F. R. Gantmacher, Matrix Theory, Vol. II, Chelsea, New York, NY, USA, 1964.

[16] Marshall Carleton Pease Methods of Matrix Algebra. Academic Press, 1965. 


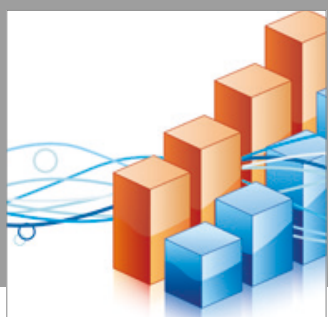

Advances in

Operations Research

vatersals

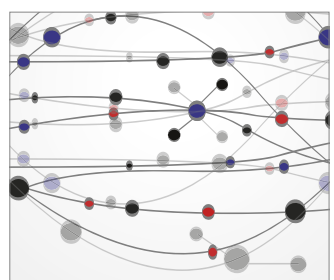

\section{The Scientific} World Journal
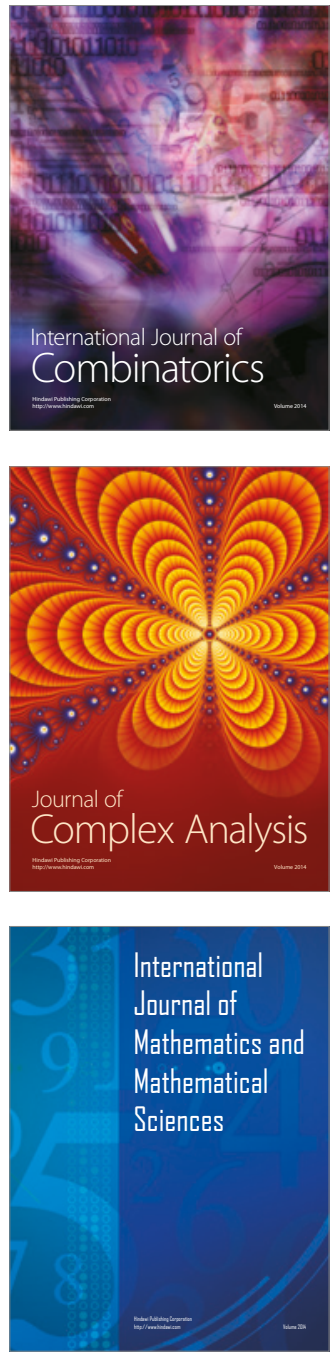
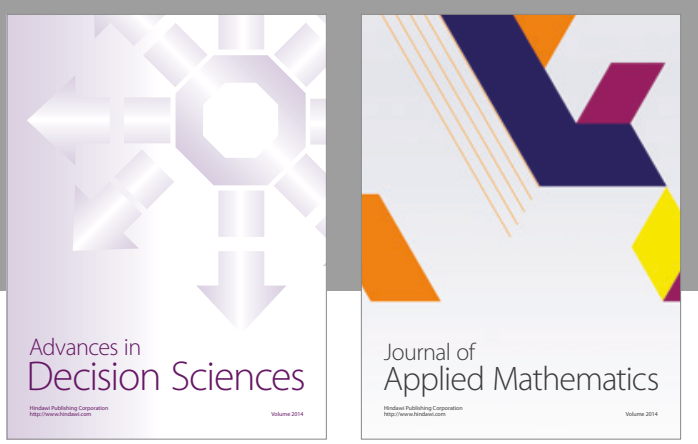

Algebra

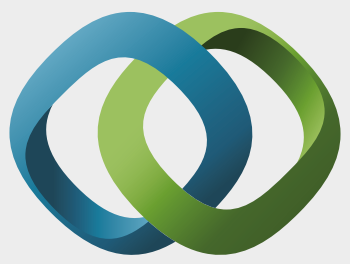

\section{Hindawi}

Submit your manuscripts at

https://www.hindawi.com
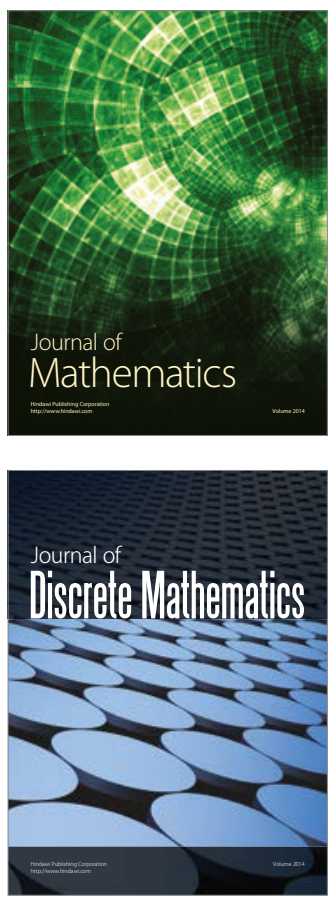

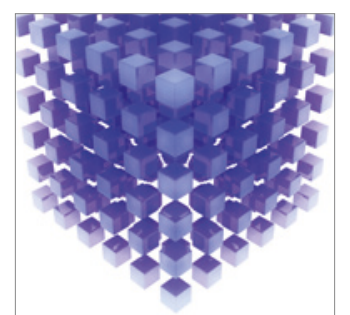

Mathematical Problems in Engineering
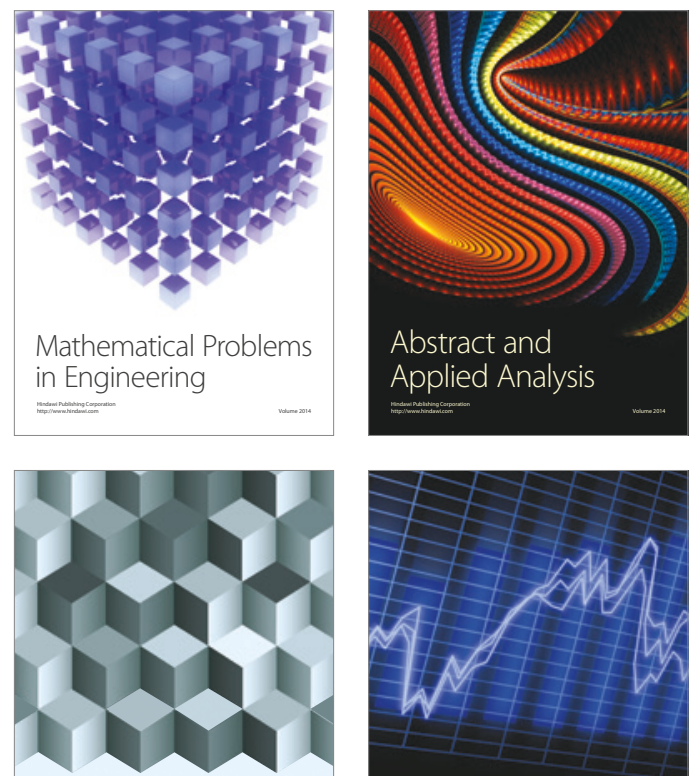

Journal of

Function Spaces

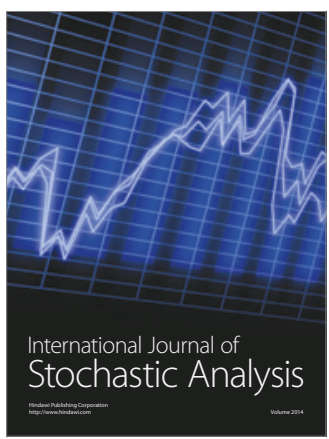

Probability and Statistics
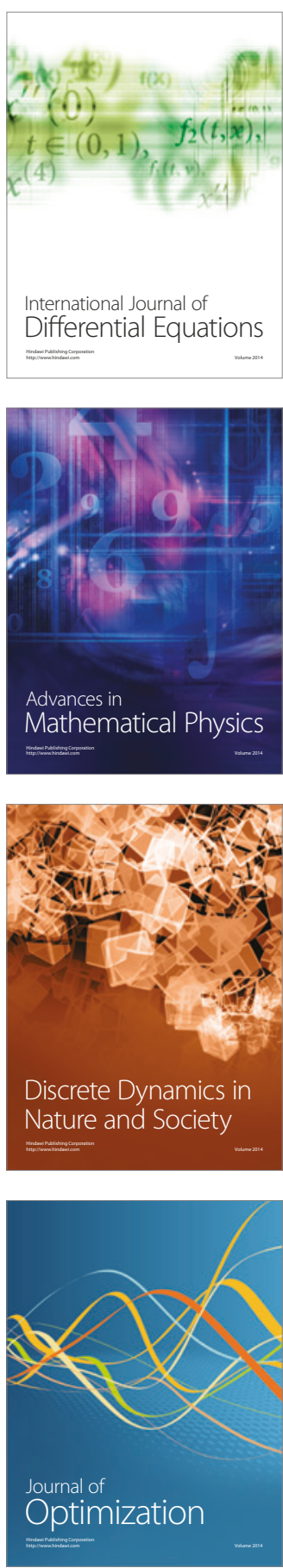